International Journal of Pure and Applied Mathematics

Volume 85 No. 3 2013, 557-562

ISSN: 1311-8080 (printed version); ISSN: 1314-3395 (on-line version)

url: http://www.ijpam.eu

doi: http://dx.doi.org/10.12732/ijpam.v85i3.10

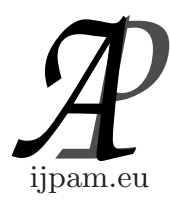

\title{
MATCHING AND EDGE COVERING NUMBER ON LEXICOGRAPHICAL PRODUCT OF COMPLETE GRAPHS
}

\author{
Thanin Sitthiwirattham \\ Department of Mathematics \\ Faculty of Applied Science \\ King Mongkut's University of Technology North Bangkok \\ Bangkok, 10800, THAILAND
}

\begin{abstract}
Let $\alpha^{\prime}(G)$ and $\beta^{\prime}(G)$ be the matching and edge covering number , respectively. The lexicographical product $G_{1} \bullet G_{2}$ of graph of $G_{1}$ and $G_{2}$ has vertex set $V\left(G_{1} \bullet G_{2}\right)=V\left(G_{1}\right) \times V\left(G_{2}\right)$ and edge set $E\left(G_{1} \bullet G_{2}\right)=$ $\left\{\left(u_{1} v_{1}\right)\left(u_{2} v_{2}\right) \mid\left[u_{1} u_{2} \in E\left(G_{1}\right)\right] \cup\left[u_{1}=u_{2}\right.\right.$ and $\left.\left.v_{1} v_{2} \in E\left(G_{2}\right)\right]\right\}$. In this paper, we determined generalization of matching and edge covering number on lexicographical product of complete graphs and any simple graph.
\end{abstract}

AMS Subject Classification: 05C69, 05C70, 05C76

Key Words: lexicographical product, matching number, edge covering number

\section{Introduction}

In this paper, graphs must be simple graphs which can be the trivial graph. Let $G_{1}$ and $G_{2}$ be graphs. The lexicographical product of graph $G_{1}$ and $G_{2}$, denote by $G_{1} \bullet G_{2}$, is the graph with $V\left(G_{1} \bullet G_{2}\right)=V\left(G_{1}\right) \times V\left(G_{2}\right)$ and $E\left(G_{1} \bullet G_{2}\right)=$ $\left\{\left(u_{1} v_{1}\right)\left(u_{2} v_{2}\right) \mid\left[u_{1} u_{2} \in E\left(G_{1}\right)\right] \cup\left[u_{1}=u_{2}\right.\right.$ and $\left.\left.v_{1} v_{2} \in E\left(G_{2}\right)\right]\right\}$. There are some properties about lexicographical product of graph. We recall these here.

Proposition 1. Let $H=G_{1} \bullet G_{2}=(V(H), E(H))$ then:

Received: February 9, 2013

(c) 2013 Academic Publications, Ltd. url: www.acadpubl.eu 
(i) $|V(H)|=\left|V\left(G_{1}\right)\right|\left|V\left(G_{2}\right)\right|$;

(ii) $|E(H)|=\left|V\left(G_{1}\right)\right|\left|V\left(G_{2}\right)\right|^{2}+\left|V\left(G_{1}\right)\right|\left|E\left(G_{2}\right)\right|$;

(iii) for every $(u, v) \in V(H), d_{H}((u, v))=2\left|V\left(G_{2}\right)\right|+d_{G_{2}}(v)$.

Theorem 2. Let $G_{1}$ and $G_{2}$ be connected graphs, The graph $H=G_{1} \bullet G_{2}$ is connected if and only if $G_{1}$ is connected .

Next we get that general form of graph of lexicographical product of $K_{n}$ and a simple graph.

Proposition 3. Let $G$ be connected graph order $m$, the graph of $K_{n} \bullet$ $G$ is

$$
\left[\left(\bigcup_{i=1}^{n-1} H_{i}\right)\right] \cup \bigcup_{i=1}^{n} R_{i} ; \quad H_{i}=\bigcup_{j=i+1}^{n} H_{i j}
$$

where $V\left(H_{i}\right)=W_{i} \cup W_{j} ; W_{i}=\{(i, 1), \ldots,(i, m)\} ; E\left(H_{i j}\right)=\{(i, v)(j, v) / v \in$ $V(G)\}$ and $V\left(R_{i}\right)=W_{i} ; E\left(R_{i}\right)=\{(i, u)(i, v) / u v \in E(G)\}$ Moreover, $H_{i j}$ isomorphic to complete bipartite graph $K_{|V(G)|,|V(G)|}$ and $R_{i}$ isomorphic to $G$.

Example

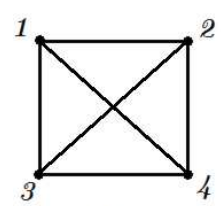

$K_{4}$

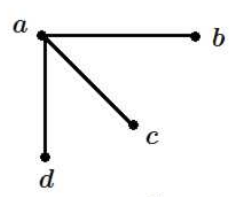

$G$

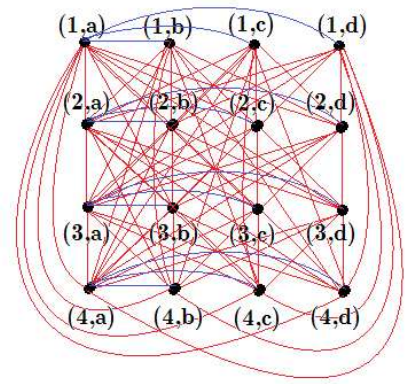

$K_{4} \cdot G$

Figure 1: The graph of $K_{4} \bullet G$

Next, we give the definitions about some graph parameters. A subset of the edge set $E$ of $G$ is said to be matching or an independent edge set of $G$, if no two distinct edges in $M$ have a common vertex. A matching $M$ is maximum matching in $G$ if there is no matching $M^{\prime}$ of $G$ with $\left|M^{\prime}\right|>|M|$. The cardinality of maximum matching of $G$ is called the matching number of $G$, denoted by $\alpha^{\prime}(G)$. 
An edge of graph $G$ is said to cover the two vertices incident with it, and an edge cover of a graph $G$ is a set of edges covering all the vertices of $G$. The minimum cardinality of an edge cover of a graph $G$ is called the edge covering number of $G$, denoted by $\beta^{\prime}(G)$.

By definitions of edge covering number and matching number, clearly that $\alpha^{\prime}\left(K_{n}\right)=\left\lfloor\frac{n}{2}\right\rfloor$ and $\beta^{\prime}\left(K_{n}\right)=\left\lceil\frac{n}{2}\right\rceil$.

\section{Matching Number of the Graph of $K_{n} \otimes G$}

We begin this section by giving the definition and theorem for alternating path and augmenting path, the lemma 6 that shows character of matching for each $H_{i j}$ and $R_{i}$.

Definition 4. Given a matching $M$, an $M$-alternating path is a path that alternates between edges in $M$ and edges not in $M$. An $M$-alternating odd path whose endpoints are unsaturated by $M$ is an $M$-augmenting path.

Theorem 5. A matching $M$ in a graph $G$ is a maximum matching in $G$ if and only if $G$ has no $M$-augmenting path.

Next, we giving the lemma 6 which show character of matching for each $H_{i j}$ and $R_{i}$.

Lemma 6. Let $K_{n} \otimes G=\left[\left(\bigcup_{i=1}^{n-1} H_{i}\right)\right] \cup \bigcup_{i=1}^{n} R_{i} ; \quad H_{i}=\bigcup_{j=i+1}^{n} H_{i j}$. Then $\alpha^{\prime}\left(H_{i j}\right)=|V(G)|$ and $\alpha^{\prime}\left(R_{i}\right)=\alpha^{\prime}(G)$.

Proof. By proposition 3 , we get $H_{i j} \cong K_{|V(G)|,|V(G)|}, R_{i} \cong G$.

Hence $\alpha^{\prime}\left(H_{i j}\right)=|V(G)|$ and $\alpha^{\prime}\left(R_{i}\right)=\alpha^{\prime}(G)$.

Next, we establish theorem 7 for a matching number of $K_{n} \otimes G$

Theorem 7. Let $G$ be connected graph order $m$, then

$$
\alpha^{\prime}\left(K_{n} \bullet G\right)= \begin{cases}\left\{\frac{m n}{2}\right\} & , n \text { is even } \\ \left\{\frac{m n}{2}+\alpha^{\prime}(G)\right\} & , n \text { is odd }\end{cases}
$$

Proof. Let $V\left(K_{n}\right)=\left\{u_{i}, i=1,2, \ldots, n\right\}, V(G)=\left\{v_{j}, j=1,2, \ldots, m\right\}, W_{i}=$ $\left\{\left(u_{i}, v_{j}\right) \in V\left(K_{n} \bullet G\right) / j=1,2, \ldots, m\right\}, \quad i=1,2, \ldots, n$. By lemma 6 , we have $\alpha^{\prime}\left(H_{i j}\right)=|V(G)|$. Since $K_{n} \bullet G$ is $\left[\bigcup_{i=1}^{n-1} H_{i}\right] \cup \bigcup_{i=1}^{n} R_{i}$ which have matching in $H_{k}$ 
be $\left\{\left(u_{k}, v_{j}\right),\left(u_{k+1}, v_{j}\right) / j=1,2, \ldots, m\right\} ; k=1,3, \ldots, 2\left\lfloor\frac{n}{2}\right\rfloor-1$ where $n$ is even. In the case $n$ is odd, we get matching in $H_{k}$ and $R_{n}$.Hence

$$
\alpha^{\prime}\left(K_{n} \bullet G\right) \geq \begin{cases}\left\{\frac{m n}{2}\right\} & , n \text { is even } \\ \left\{\frac{m n}{2}+\alpha^{\prime}(G)\right\} & , n \text { is odd }\end{cases}
$$

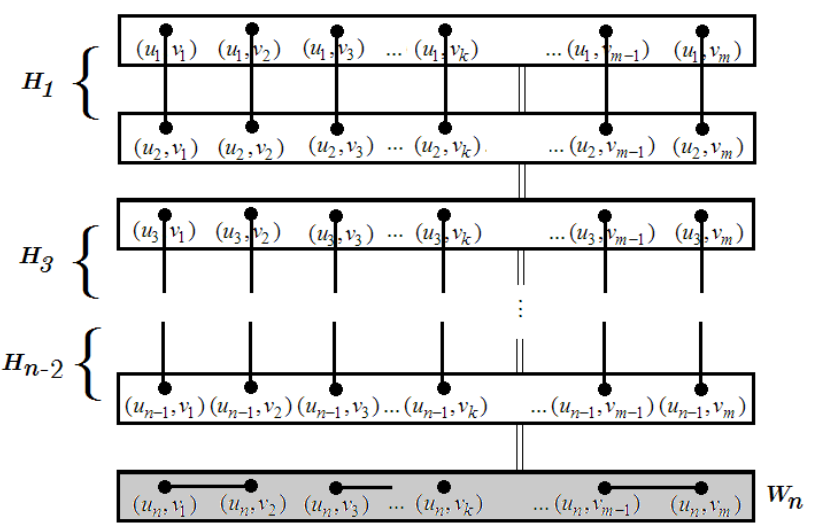

Figure 2: The matching when $n$ is odd, $m$ is even

In the case $n$ is even, if $\alpha^{\prime}\left(K_{n} \bullet G\right)>\frac{m n}{2}$. It is impossible because every vertices of $K_{n} \bullet G$ are matching out already.

In the case $n$ is odd, we let $M$ is maximum matching of $G$. If $\alpha^{\prime}\left(K_{n} \bullet G\right)>$ $\frac{m n}{2}+\alpha^{\prime}(G)$, then there exist a matching $M$ is augmenting path. That is not true because each vertices in $K_{n} \bullet G$ always incident with edges in $M$ and another edges which are not in $M$.

Hence

$$
\alpha^{\prime}\left(K_{n} \bullet G\right)= \begin{cases}\left\{\frac{m n}{2}\right\} & , n \text { is even } \\ \left\{\frac{m n}{2}+\alpha^{\prime}(G)\right\} & , n \text { is odd }\end{cases}
$$

\section{Edge Covering Number of the Graph of $K_{n} \otimes G$}

We begin this section by giving the lemma 8 that shows a relation of matching number and edge covering number and the lemma 9 that show character of edge cover number for each $H_{i}$. 
Lemma 8. Let $G$ be a simple graph with order $n$. Then $\alpha^{\prime}(G)+\beta^{\prime}(G)=n$ Next we establish theorem 9 for a edge covering number of $K_{n} \bullet G$.

Theorem 9. Let $G$ be connected graph of order $m$, then

$$
\alpha^{\prime}\left(K_{n} \bullet G\right)= \begin{cases}\left\{\frac{m n}{2}\right\} & , n \text { is even } \\ \left\{\beta^{\prime}(G)-\frac{m n}{2}\right\} & , n \text { is odd }\end{cases}
$$

Proof. By theorem 7 and lemma 8, we can also show that

$$
\begin{aligned}
\alpha^{\prime}\left(K_{n} \bullet G\right)+\beta^{\prime}\left(K_{n} \bullet G\right) & =m n \\
\frac{m n}{2}+\beta^{\prime}\left(K_{n} \bullet G\right) & =m n \\
\beta^{\prime}\left(K_{n} \bullet G\right) & =\frac{m n}{2}, n \text { is even. }
\end{aligned}
$$

and,

$$
\begin{aligned}
\alpha^{\prime}\left(K_{n} \bullet G\right)+\beta^{\prime}\left(K_{n} \bullet G\right) & =m n \\
{\left[\frac{m n}{2}+\alpha^{\prime}(G)\right]+\beta^{\prime}\left(K_{n} \bullet G\right) } & =m n \\
\beta^{\prime}\left(K_{n} \bullet G\right) & =m n-\frac{m n}{2}-\alpha^{\prime}(G) \\
& =\beta^{\prime}(G)-\frac{m n}{2}, n \text { is odd }
\end{aligned}
$$

Hence

$$
\alpha^{\prime}\left(K_{n} \bullet G\right)= \begin{cases}\left\{\frac{m n}{2}\right\} & , n \text { is even } \\ \left\{\beta^{\prime}(G)-\frac{m n}{2}\right\} & , n \text { is odd }\end{cases}
$$

\section{References}

[1] B.W. Douglus, Introduction to Graph Theory, Prentice-Hall (2001).

[2] P.M. Weichsel, The Kronecker product of graphs, Proc. Amer. Math. Soc., 8 (1962), 47-52.

[3] T. Sitthiwirattham, J. Soontraranon, Independent and vertex covering number on Kronecker product of Cn, IJPAM, 71, No. 1 (2011), 149-157.

[4] Z.A. Bottreou, Y. Metivier, Some remarks on the Kronecker product of graph, Inform. Process. Lett, 8 (1998), 279-286. 
[5] T. Sitthiwirattham, Edge covering and matching number on Kronecker product of Kn, Applied Mathematical Sciences, 6, No. 28 (2012), 19371402. 